\title{
New silicone tipped cannulas for subretinal fluid drainage
}

\author{
W SANDERSON GRIZZARD' AND LIAQUAT ALLARAKHIA²
}

From the 'Department of Ophthalmology, University of South Florida, College of Medicine, Tampa, Florida, and ${ }^{2}$ Research and Development, Visitec Company, Sarasota, Florida, USA

SUMMARY Two new disposable cannulas for internal drainage of subretinal fluid are described in this article. We describe a curved and a straight version consisting of plastic hubs, 20 gauge proximal and 25 gauge distal stainless steel hypodermic tubing, and soft and flexible silicone tubing overmounts. Both these cannulas facilitate atraumatic drainage of subretinal fluid through preexisting retinal holes and tears or through a retinotomy performed during vitreoretinal microsurgery.

Vitreoretinal microsurgery is routinely performed for the correction of complex retinal detachment, and there is an increasing trend of performing total intraocular treatment, including vitrectomy, fluidgas exchange, and endophotocoagulation. To maintain this form of total intraocular treatment it is better to perform internal drainage of subretinal fluid than external drainage, which may often be difficult, cumbersome, and time consuming. Flynn et al' have developed a sophisticated cannulated extrusion needle for this purpose. However, this is an expensive and reusable device and can be used only in combination with a complex fluid-gas exchange machine. Flynn et $a l^{2}$ have also described an inexpensive straight, silicone tipped extrusion cannula which can be fashioned by the surgeon prior to surgical use.

We have developed two separate cannulas, one straight, the other curved. Each cannula has a yellow, colour coded plastic hub attached to hypodermic stainless steel tubing. The metal tube is $28 \mathrm{~mm}$ long, with the 20 gauge proximal segment being $22 \mathrm{~mm}$ long and the distal 25 gauge segment being $6 \mathrm{~mm}$. A medical grade silicone tube, $12 \mathrm{~mm}$ long, is mounted over the distal metal segment thus enabling a 'silicone only' protrusion of approximately $6 \mathrm{~mm}$. The curved version has a distal segment radius of curvature of approximately $10 \mathrm{~mm}$. The curvature is minimal and facilitates entry into holes or tears that are difficult to reach.

The cannulas are commercially manufactured and

Correspondence to Liaquat Allarakhia, MD, 7575 Commerce Covet, Sarasota. Florida 34243, USA therefore have consistent, smooth, well rounded edges, and securely mounted silicone tubing overmounts that will not inadvertently dislodge into the vitreous cavity or in the subretinal space during the drainage procedure. The external surface of the terminal segment of the metal cannula is vapour blasted to facilitate a secure friction fit of the silicone overmount.

The soft and extremely flexible silicone tip of either

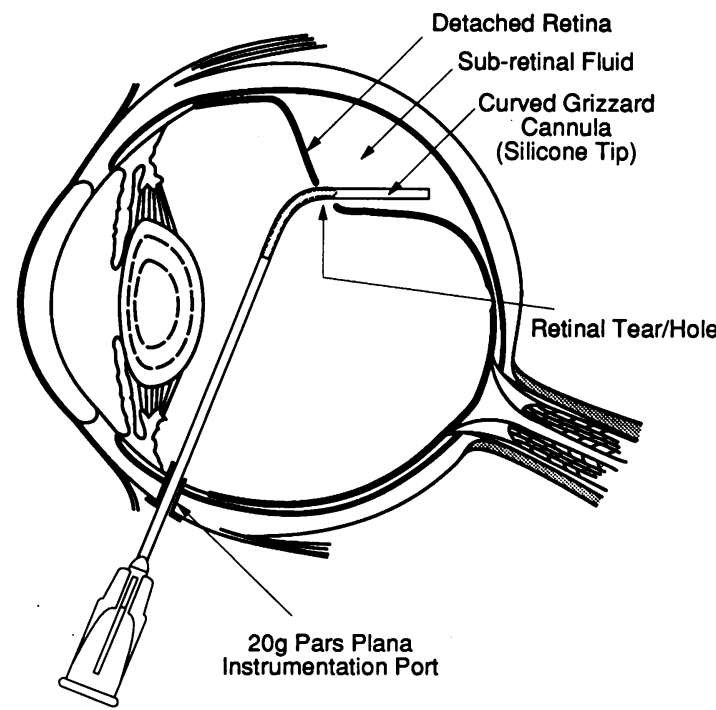

Fig. 1 Schematic depiction of curved cannula in use during internal drainage of subretinal fluid. 
cannula can be inserted into an existing posterior or peripheral retinal break or hole and guided into the subretinal space (Fig. 1). These cannulas can similarly be used in situations where the surgeon needs to make a hole to facilitate internal drainage. The cannulas can be used by being attached to a flute handle or alternatively in conjunction with an automated suction device. The main shaft of both cannulas is 20 gauge and therefore can be inserted through a 20 gauge incision or instrumentation port. The straight cannula is ideal for posterior tears, and the curved design enables easier manipulation through peripheral tears. When the retinal hole or break is small, the silicone overmount can be removed to facilitate insertion of the 25 gauge distal segment of the metal cannula through this small hole. The silicone tipped cannulas described in this article are available from Visitec Company, Sarasota, Florida, USA.

The authors have no financial interest in the cannulas described in this article.

\section{References}

1 Flynn HW, Davies JL. Parel J-M. Lee WG. Applications of a cannulated extrusion needle during vitreoretinal microsurgery. Retina 1988; 8: 42-9.

2 Flynn HW, Lee WG, Parel J-M. A simple extrusion needle with flexible cannula tip for vitreoretinal microsurgery. $A m J$ Ophthalmol 1988: 105: 215-6.

Accepted for publication 17 April 1989 